\title{
Influence of YKL-40 gene RNA interference on the biological behaviors of endometrial cancer HEC-1A cells
}

\author{
LILI LI, JIANGTAO FAN, DAHAI LI, YAN LIU, POONAM SHRESTHA, CHUNYAN ZHONG, \\ XIUHONG XIA and XIAOBING HUANG
}

Department of Gynecology, The First Affiliated Hospital of Guangxi Medical University, Nanning, Guangxi 530021, P.R. China

Received December 25, 2016; Accepted October 13, 2017

DOI: $10.3892 / 01.2018 .8814$

\begin{abstract}
The present study aimed to investigate the effects of chitinase-3-like protein 1 (YKL-40) gene RNA interference on the biological behaviors and enhanced chemosensitivity of endometrial cancer (EC) HEC-1A cells. YKL-40 small interfering (si)RNA was transduced into EC HEC-1A cells using a lentivirus. The experiment was divided into three groups: The experimental group was transfected with YKL-40 siRNA (si-YKL-40); the mock-treatment group was transfected with transfection reagent only; and the blank control group was left untreated. A reverse transcription-quantitative polymerase chain reaction was performed to investigate the mRNA expression levels of YKL-40. The biological behaviors, including cell proliferation, migration, invasion and apoptosis, were detected by MTT and Transwell assays, and flow cytometry (FCM) analysis, respectively. The results of the present study demonstrated that the mRNA expression levels of YKL-40 were downregulated within HEC-1A cells upon transfection with si-YKL-40 $(\mathrm{P}<0.05)$. The proliferative, migratory and invasive abilities of HEC-1A cells were inhibited by si-YKL-40 $(\mathrm{P}<0.05)$. The mRNA expression levels of YKL-40 were upregulated within HEC-1A cells following treatment with cisplatin $(\mathrm{P}<0.05)$. FCM analysis revealed that the average cellular apoptosis rate increased following the inhibition of YKL-40 gene expression via siRNA $(\mathrm{P}<0.05)$. Therefore, the YKL-40 gene may be associated with the proliferative, migratory, invasive and anti-apoptotic ability
\end{abstract}

Correspondence to: Professor Jiangtao Fan, Department of Gynecology, The First Affiliated Hospital of Guangxi Medical University, 6 Shuangyong Road, Nanning, Guangxi 530021, P.R. China

E-mail: jiangtao_fan@163.com

Abbreviations: YKL-40, chitinase-3-like protein 1; siRNA, small interfering RNA; FCM, flow cytometry; EC, endometrial cancer; DMEM, Dulbecco's modified Eagle's medium; FBS, fetal bovine serum; OD, optical density; 7AAD, 7-actinomycin D; PE, phycoerythrin; ERK, extracellular signal-regulated kinase

Key words: YKL-40, siRNA, EC, biological behaviors, cisplatin, chemotherapy of HEC-1A cells. YKL-40 downregulation may enhance the sensitivity of human EC HEC-1A cells to chemotherapy.

\section{Introduction}

Endometrial cancer (EC) is one of the most malignant forms of cancer of the female reproductive system. Chemotherapy combined with surgery and radiotherapy is the most important method of contemporary EC treatment. Previously, studies demonstrated that YKL-40 is one of the candidate biomarkers of EC; the YKL-40 gene may serve an important role in the proliferation (1), angiogenesis (2), and anti-apoptosis (3) of cancer cells. However, the function of YKL-40 requires further investigation. A previous study (4) reported that the YKL-40 protein is highly expressed in a variety of tissues and in the serum of patients with EC, compared with patients with uterine myoma and healthy individuals.

In the present study, a unique small interfering (si)RNA sequence was used to prepare a recombinant lentivirus which was transduced into tumor cells and was effective in inhibiting the expression of the YKL-40 gene. MTT, migration, invasion and flow cytometry (FCM) assays were performed to identify the effects of si-YKL-40 on the proliferative, migratory and invasive abilities, and apoptotic rate, of EC HEC-1A cells. FCM analysis of the average cellular apoptotic rate following treatment with cisplatin, pre- and post-si-YKL-40 transduction, was used to understand the effects of si-YKL-40 on the sensitivity of EC HEC-1A cells to cisplatin-based chemotherapy. Additionally, further investigation is required to understand the underlying mechanism of EC chemoresistance. Combining YKL-40 siRNA with other chemotherapies may provide efficacious therapy for the treatment of cancer. Targeting the YLK-40 gene may control the occurrence of EC development in the future.

\section{Materials and methods}

Cell lines and cell culture. HEC-1A cells were obtained from the Affiliated Tumor Hospital of Guangxi Medical University (Nanning, China). The cells were maintained at $37^{\circ} \mathrm{C}$ in a humidified incubator under $5 \% \mathrm{CO}_{2}$ and cultured in Dulbecco's modified Eagle's medium (DMEM)/F12 culture medium (GE Healthcare, Chicago, IL, USA) supplemented with $10 \%$ fetal bovine serum (FBS; Gibco; Thermo Fisher 
Scientific, Inc., Waltham, MA, USA), $100 \mathrm{U} / \mathrm{ml}$ penicillin, and $100 \mu \mathrm{g} / \mathrm{ml}$ streptomycin. The cells were subcultured every 2 days. Cells in the logarithmic growth phase were used for the experiments.

YKL-40 siRNA sequences. A DNA oligomer specifically targeting the sequence of YKL-40 5'-GACTCTCTTTCT GTCGGA-3' (si-YKL-40; Hanbio Biotechnology Co., Ltd., Shanghai, China) was selected and subcloned into a retroviral pSUPER-puro-vector (Hanbio Biotechnology Co., Ltd.). Subsequently, 293T retroviral packaging cells (American Type Culture Collection, Manassas, VA, USA) were transfected with the si-YKL-40-pSUPER puro-vector $\left(1 \times 10^{8} / \mathrm{ml}\right.$; Hanbio Biotechnology Co., Ltd.). After $48 \mathrm{~h}$ post-transfection, the supernatant which was centrifuged at $72,000 \mathrm{x} \mathrm{g} / \mathrm{min}$ was harvested and filtered in $4^{\circ} \mathrm{C}$ for $120 \mathrm{~min}$ through a $0.45 \mu \mathrm{m}$ pore-size filter. All of the transfected reagents were purchased from Hanbio Biotechnology Co., Ltd.

Cell transfection and knockdown of YKL-40 within HEC-1A cells. HEC-1A cells were divided into three groups: The experimental group was transfected with siRNA, the mock-treatment group was treated with transfection reagent only, and the blank control group was left untransfected. HEC-1A ( $5 \times 10^{4}$ cells) were transferred into 6 -well plates, then transfected when cells reached $50 \%$ confluence. The results of a previous study (5) indicated that the optimal multiplicity of infection value was 20 ; therefore, this number was selected to transfect the cells (si-YKL-40; Hanbio Biotechnology Co., Ltd.). Cells were transfected with si-YKL-40 or nontargeting green fluorescence protein (GFP; Hanbio Biotechnology Co., Ltd.) in the presence of Lipofectamine 2000 (Invitrogen; Thermo Fisher Scientific, Inc.) according to the manufacturer's protocol. The cells that were successfully transfected exhibited GFP. Following transfection, a lentivirus was used to transduce HEC-1A cells. Selection with $1 \mu \mathrm{g} / \mathrm{ml}$ puromycin was performed for 2 weeks following transduction; puromycin-resistant cells were used for subsequent experiments.

Detection of $Y K L-40$ expression by reverse transcription-quantitative polymerase chain reaction (RT-qPCR). Total RNA was extracted using TRIzol reagent (Sigma-Aldrich; Merck KGaA, Darmstadt, Germany), according to the manufacturer's protocol. The optical density (OD) 260/OD280 ratio for determining the purity of RNA was reported to be between 1.8-2.0. A total of $1 \mu \mathrm{g}$ of RNA was reverse transcribed using the first cDNA strand (cDNA synthesis kit; Takara Bio, Inc., Otsu, Japan). RT-qPCR was performed using an ABI StepOne Plus (Applied Biosystems; Thermo Fisher Scientific, Inc.) system with SYBR Green PCR Master Mix (Takara Bio, Inc.). RT-qPCR reactions were performed according to the manufacturers' protocols. RT-qPCR assays were performed in triplicate, and the mean values were used to calculate mRNA expression. Gene expression was normalized to $\beta$-actin mRNA. The results were calculated and presented as $2^{-\Delta \Delta C q}(6)$. The primer sequences were as follows: YKL-40, 5'-ATCACCAAGGAGCCAAAC ATC-3' (sense) and 5'-GGGGAAGTAGGATAGGGGACA-3' (antisense); $\beta$-actin, 5'-ACACTGTGCCCATCTACG-3' (sense) and 5'-TGTCACGCACGATTTCC-3' (antisense).
Cell proliferation assays. For the proliferation assays, cells $\left(5 \times 10^{3}\right)$ were seeded onto $96-w e l l$ plates with $100 \mu \mathrm{lDMEM} / \mathrm{F} 12$ medium and incubated overnight at $37^{\circ} \mathrm{C}$. Five replicates were prepared for each cell type. Cells from each of the groups were cultured for 24, 48, 72, 96 and $108 \mathrm{~h}$. To measure proliferation, MTT reagent $[20 \mu 15 \mathrm{mg} / \mathrm{ml}$ thiazolyl blue tetrazolium bromide in PBS (Sigma-Aldrich; Merck KGaA)] was added into each well and incubated for $4 \mathrm{~h}$. The reaction was stopped by adding $150 \mu 1$ dimethyl sulfoxide to each well. The OD value of blue emission was analyzed with a microplate reader (Thermo Fisher Scientific, Inc.) at a $570 \mathrm{~nm}$ wavelength. The cell growth curve was determined using OD values as the ordinate and duration as the abscissa. All the experiments were conducted in triplicate.

Cell migration assays and Matrigel invasion assays. Cells $\left(1 \times 10^{4}\right)$ were seeded onto Costar Transwells (polycarbonate membrane, 24-well format, $0.8 \mathrm{~mm}$ pore size; Corning Incorporated, Corning, NY, USA) over the upper chamber coated with $60 \mu \mathrm{l}$ Matrigel (1:7; BD Biosciences, San Jose, CA, USA) which was diluted with serum-free DMEM/F12. This was followed by the addition of $500 \mu \mathrm{l}$ DMEM/F12 containing $10 \% \mathrm{FBS}$ at the bottom. The cells were incubated for $48 \mathrm{~h}$ at $37^{\circ} \mathrm{C}$. The cells in the upper chamber were wiped with wet wipes, while the cells in the bottom were washed with PBS. Subsequently, the cells were fixed with $4 \%$ paraformaldehyde for $20 \mathrm{~min}$ at room temperature and stained with $0.1 \%$ crystal violet for $30 \mathrm{~min}$ at room temperature. Average cell numbers were calculated from five different fields (magnification, x20) in each sample by an light inverted microscope (CKX41SF; Olympus Corporation, Tokyo, Japan). In addition, cells required incubation for only $24 \mathrm{~h}$ in the cell migration assay. The experiments were repeated three times.

Effect of cisplatin on the expression of YKL-40 mRNA within $H E C-1 A$ cells. HEC-1A cells which were not transduced with si-YKL-40 were divided into two groups constituting untreated cells (group A) and those treated with $25 \mathrm{mmol} / \mathrm{l}$ cisplatin for $48 \mathrm{~h}$ (group B). RT-qPCR analysis was performed to investigate the mRNA levels of YKL-40 within HEC-1A cells. The experiment was performed in accordance with the aforementioned protocols. The experiments were conducted in triplicate.

MTT assays and apoptotic assays. Apoptotic assays of the transduced HEC-1A cells treated with cisplatin were performed by detecting 7-actinomycin $\mathrm{D}$ (7AAD) and Annexin V-phycoerythrin (PE) in these cells. The cells were treated with $25 \mu \mathrm{mol} / \mathrm{l}$ cisplatin for $48 \mathrm{~h}$. Cells treated with normal saline were used as the control. Cisplatin was purchased from Shandong Qilu King-Phar Pharmaceutical Co., Ltd. (Jinan, China). Preparation of the drug was performed according to the manufacturer's protocols; a concentration gradient of cisplatin $(0,50,25,12.5,6.25,3.125$, and $100 \mu \mathrm{mol} / \mathrm{l}$ ) was produced. The dosage of the cisplatin was administered according to the results of the MTT assay; as the proliferative ability of the experimental group cells was significantly inhibited compared with the blank control group cells and the mock-treatment group cells when treated with $25 \mu \mathrm{mol} / \mathrm{l}$ cisplatin. At this concentration, the chemosensitivity 

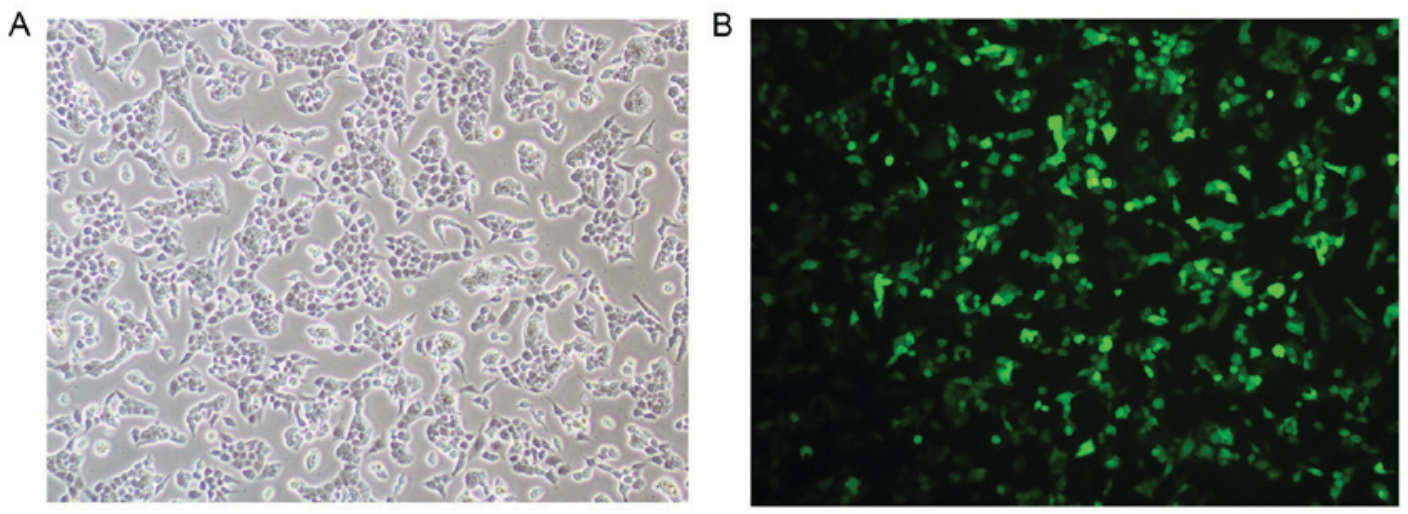

Figure 1. Detection of transfected cells (magnification, x10). Small interfering RNA was successfully transduced into the HEC-1A cells via a lentivirus. (A) HEC-1A cells were observed under bright field microscopy. (B) On the same field, HEC-1A cells were observed under a fluorescence microscope.

of HCE-1A cells to cisplatin was increased following silencing of the YKL-40 gene. The cells $\left(1 \times 10^{6} / \mathrm{ml}\right)$ were incubated with 7-AAD and PE-conjugated Annexin V (BD Biosciences) for $15 \mathrm{~min}$ at $37^{\circ} \mathrm{C}$, respectively, according to the manufacturer's protocol, and were analyzed by FCM (FACSCalibur and CellQuest software version 5.2.1; BD Biosciences).

Statistical analysis. Statistical analysis was performed using SPSS Statistics for Windows, version 17.0 (SPSS Inc., Chicago, USA). Data are presented as the mean \pm standard deviation, and significance was determined by one-way analysis of variance (ANOVA) and two independent sample t-test. The Student-Newman-Keuls and Least Significant Difference analysis were used as post-hoc tests following ANOVA to determine the difference between specific groups. $\mathrm{P}<0.05$ was considered to indicate a statistically significant difference.

\section{Results}

Detection of fluorescence in transfected cells. Treated cells were observed under a microscope. HEC-1A cells that were successfully transfected with siRNA exhibited green fluorescence (Fig. 1).

Interference effects of $Y K L-40$ siRNA. Following lentivirus-mediated transduction, the RNA transcription levels of YKL-40 within HEC-1A cells were lower compared with in the blank control and mock-treatment groups $(\mathrm{F}=6.875$; $\mathrm{P}=0.015$ ). No significant difference was observed between the blank control group and mock-treatment group $(\mathrm{P}<0.05$; Fig. 2).

YKL-40 gene controls the proliferation potential of HEC-1A cells. MTT assays indicated that si-YKL-40 inhibited HEC-1A cell proliferation compared with the blank control and mock-treatment groups $(\mathrm{P}<0.05$; Fig. 3$)$.

YKL-40 gene promotes the migratory potential of HEC-1A cells. Cell migration assays demonstrated that si-YKL-40 (133 \pm 14 migrated cells) inhibited the HEC-1A cell migration abilities compared with in the blank control $(179 \pm 19$ migrated cells) and mock-treatment groups $(178 \pm 11$ migrated cells; $\mathrm{F}=14.494 ; \mathrm{P}<0.05 ;$ Fig. 4).

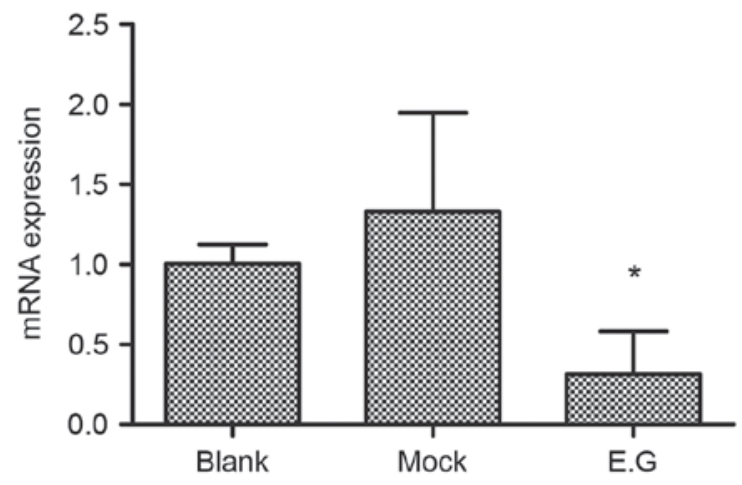

Figure 2. Relative expression of YKL-40 mRNA detected by reverse transcription-quantitative polymerase chain reaction. Following transduction with si-YKL-40, YKL-40 mRNA expression levels were significantly reduced. "P $<0.05$ vs. Mock. si-YKL-40, chitinase-3-like protein 1 small interfering RNA; E.G, experiment group; Blank, blank control group; Mock, mock-treatment group.

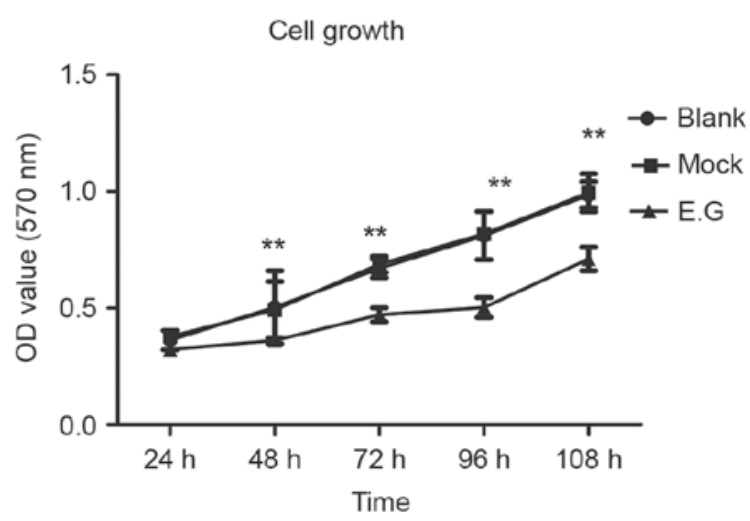

Figure 3. MTT assays in Blank, Mock and E.G cells. Chitinase-3-like protein 1 small interfering RNA inhibited HEC-1A cell proliferation. ${ }^{* *} \mathrm{P}<0.01$ vs. E.G. E.G, experiment group; Blank, blank control group; Mock, mock-treatment group; OD, optical density.

$Y K L-40$ gene regulates the invasive ability of $H E C-1 A$ cells. Matrigel invasion assays revealed that the invasive ability of HEC-1A cells within the experimental group was significantly inhibited; the invasion number $(143 \pm 13)$ was lower compared with the blank control $(227 \pm 18)$ and mock-treatment groups (238 $\pm 26 ; \mathrm{F}=33.476 ; \mathrm{P}<0.05$; Fig. 5). 
A
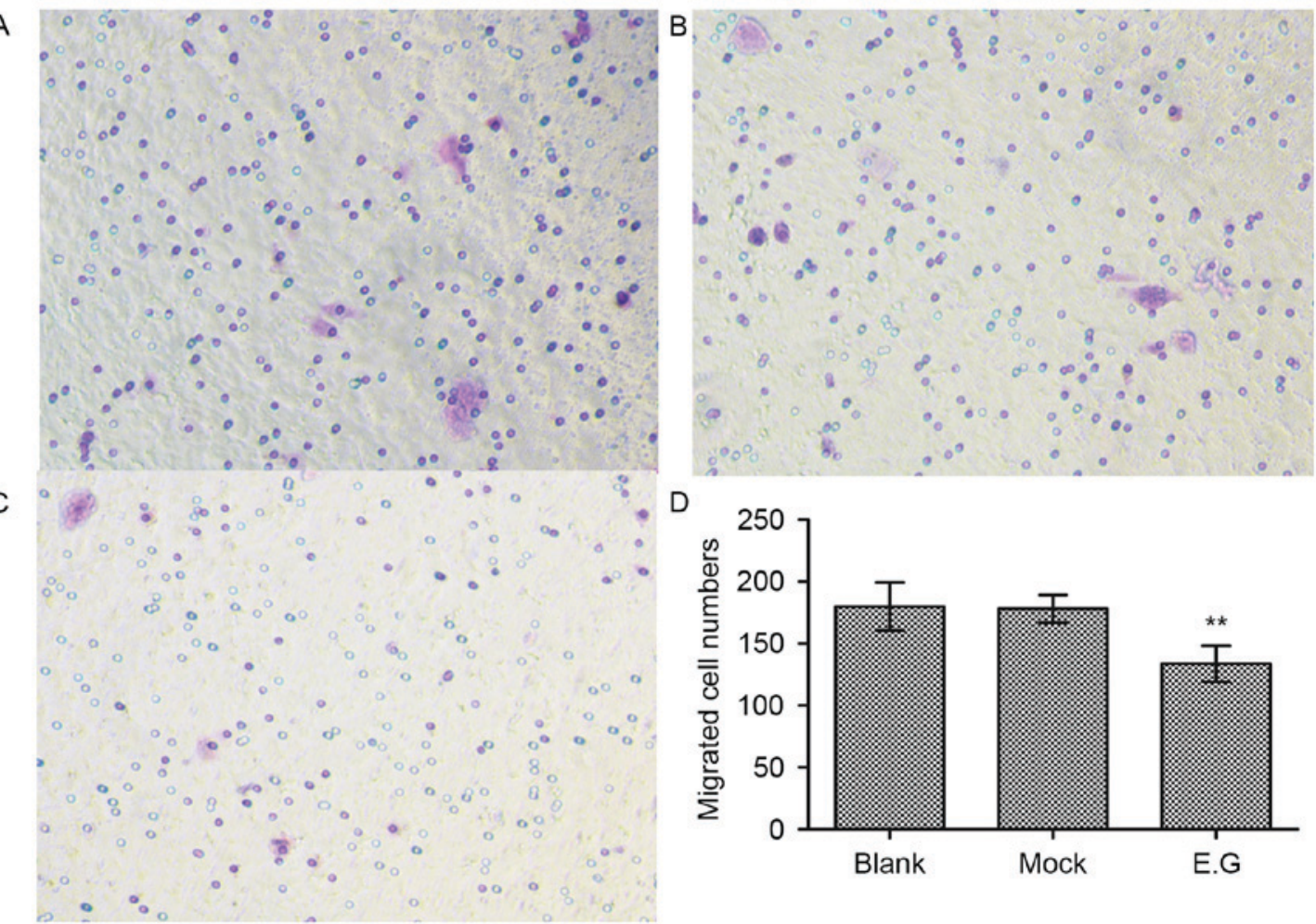

Figure 4. Chitinase-3-like protein 1-targeting small interfering RNA reduces the migration of HEC-1A cells. Cell migration assays were performed to compare the migratory ability of Blank, Mock and E.G HEC-1A cells. (A) Blank, (B) Mock and (C) E.G cells (magnification, x20). (D) Migrated cell numbers in each group. ${ }^{* *} \mathrm{P}<0.01$ vs. Mock. E.G, experiment group; Blank, blank control group; Mock, mock-treatment group.
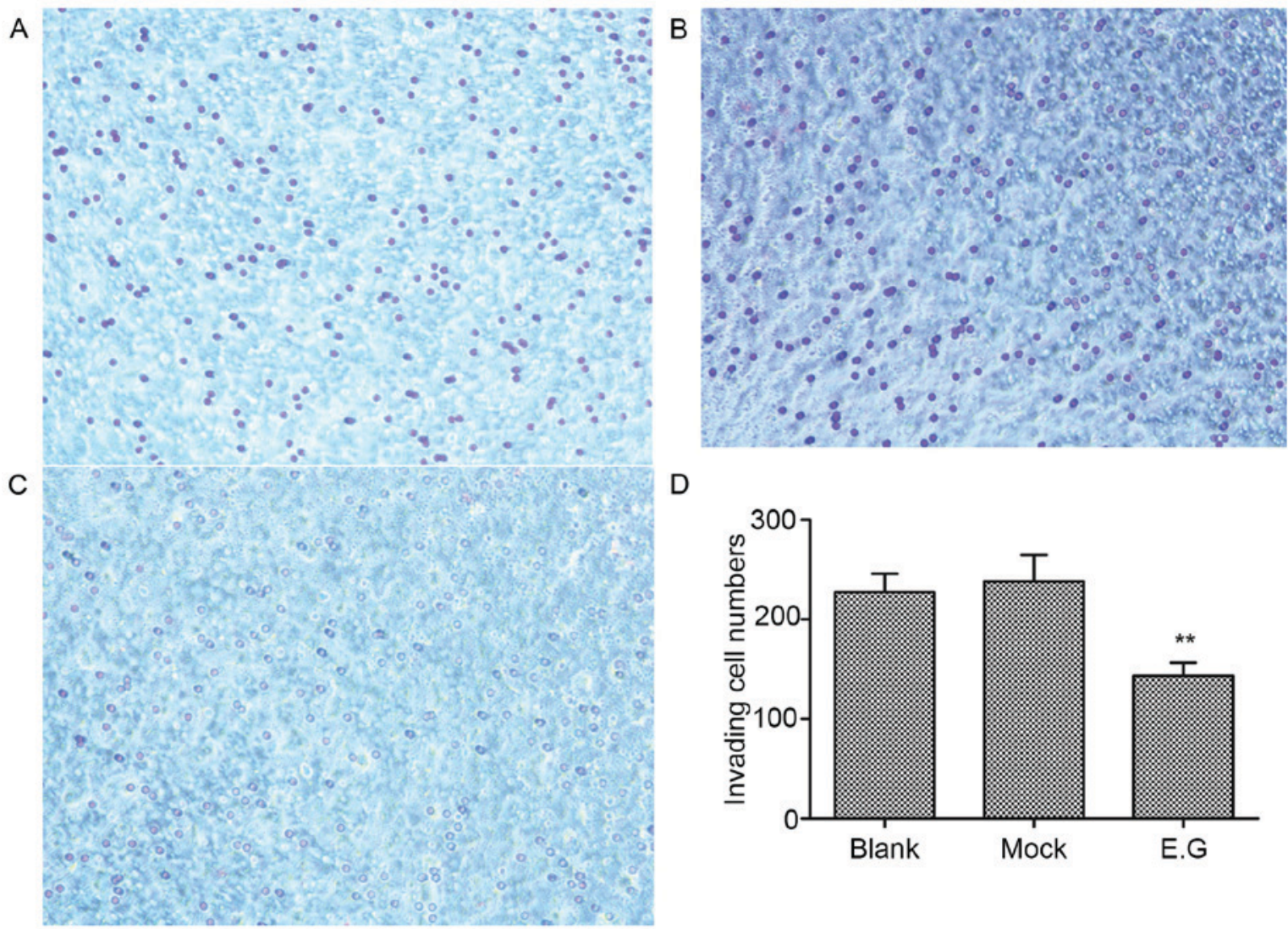

D

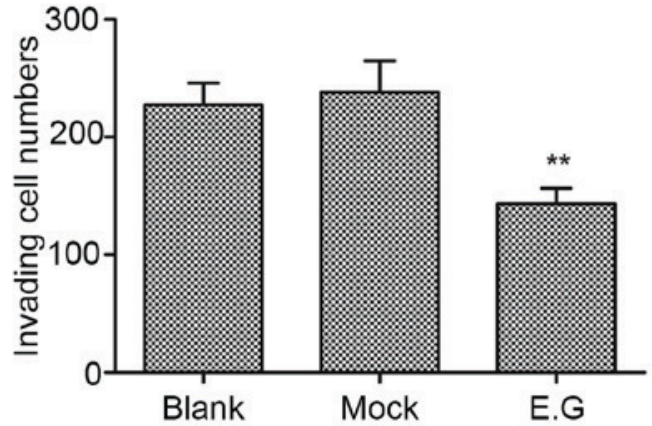

Figure 5. YKL-40 gene controls HEC-1A cell invasive ability. YKL-40 small interfering RNA reduced the invasion of HEC-1A cells. HEC-1A cells were seeded in the upper Matrigel-coated Transwell chamber, and invasion was measured by counting the number of the cells that penetrated through the artificial basal membrane. (A) Blank, (B) Mock and (C) E.G cells (magnification, x20). (D) Invading numbers of cells in each group. ${ }^{* *}$ P $<0.01$ vs. Mock. E.G, experiment group; Blank, blank control group; Mock, mock-treatment group; YKL-40, chitinase-3-like protein 1. 


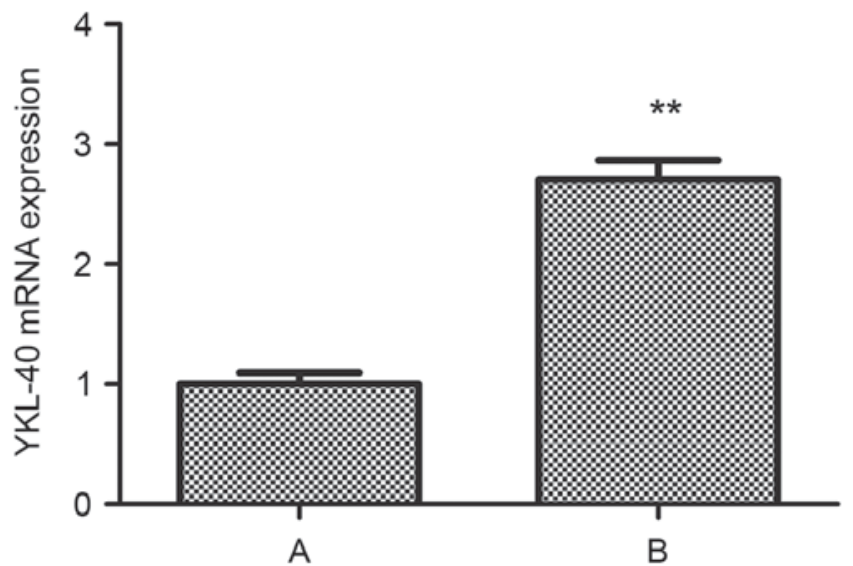

Figure 6. Relative expression of YKL- 40 mRNA detected by reverse transcription-quantitative polymerase chain reaction. YKL-40 mRNA was significantly upregulated within group B post-cisplatin treatment, compared with in group A. ${ }^{* *} \mathrm{P}<0.01$ vs. A. Data are presented as the mean \pm standard deviation. A, blank cells; B, cells treated with cisplatin. YKL-40, chitinase-3-like protein 1.

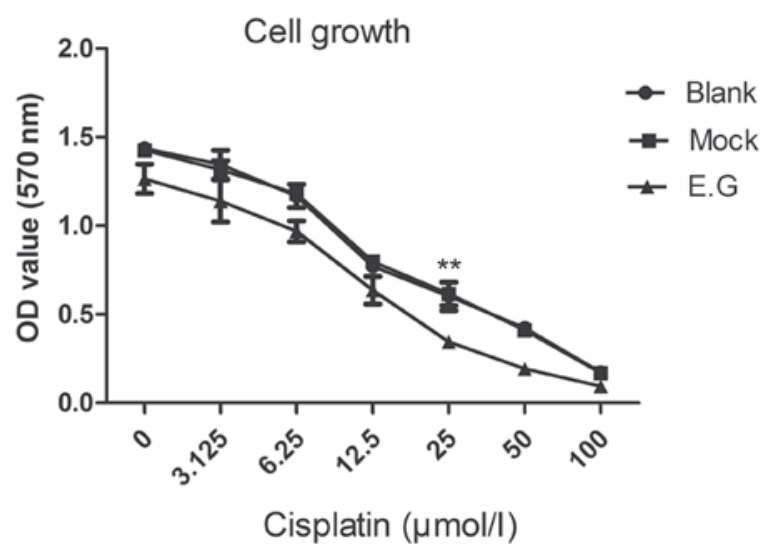

Figure 7. MTT assays of Blank, Mock and E.G cells. Chitinase-3-like protein 1 small interfering RNA inhibited HEC-1A cell proliferation when treated with cisplatin for 48 h. ${ }^{* *} \mathrm{P}<0.01$ vs. E.G. E.G, experiment group; Blank, blank control group; Mock, mock-treatment group; OD, optical density.

Cisplatin upregulates the expression of YKL-40 mRNA in human HEC-1A cells. Following treatment with cisplatin, the RNA transcription levels of YKL-40 within HEC-1A cells of group B was upregulated compared with in group A ( $\mathrm{t}=-15.986$, $\mathrm{P}<0.05$; Fig. 6).

YKL-40 gene inhibits the apoptosis of HEC-1A cells following treatment with cisplatin. YKL-40 inhibited the apoptosis of human EC cells treated with a cytotoxic drug. To investigate the possible biological effects of the YKL-40 gene on EC cells, YKL-40-transfected HEC-1A cells were generated for in vitro apoptosis assays. The HEC-1A cell apoptosis rate increased following the inhibition of YKL-40 expression (experimental

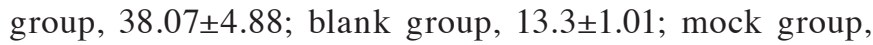
12.5 \pm 0.17 ), when treated with cisplatin for $48 \mathrm{~h}$ (Fig. 7). Following the downregulation of YKL-40 expression levels via YKL-40 siRNA, cisplatin chemotherapeutic sensitivity and the apoptosis of EC cells increased. However, there was no significant difference observed between the blank control and mock-treatment groups. The representative figures of the FCM analysis for the detection of Annexin V-positive and 7AAD-positive cells in transduced HEC-1A cells treated with cisplatin are presented in Fig. 8.

\section{Discussion}

$\mathrm{EC}$ is now the most common gynecologic malignancy, which more commonly occurs in postmenopausal women. EC consists of a group of endometrial epithelial malignant tumors with a number of pathological types, including endometrial adenocarcinoma (7). Studies have revealed that the pathogenesis of EC is associated with numerous factors (8). Clinical characterization of the type of disease is an essential step for correct diagnosis and treatment. However, the cause of EC requires further investigation. Type I EC develops in an environment of high levels of estrogen and frequently develops from endometrial hyperplasia, whereas type II cancer is not an estrogen-associated cancer, occurring predominantly in postmenopausal women. An excessive increase in estrogen may promote the accumulation of body fat, and obesity-associated proteins may promote the proliferation of endometrial carcinoma (9).

YKL-40, additionally termed CHI3L1, is produced by a variety of cells (10), including macrophages, neutrophils, eosinophils, vascular smooth muscle cells, endothelial cells and cancer cells. Elevated levels of serum YKL-40 protein have been reported in a number of other types of cancer, including glioma (11) cholangiocarcinoma (12), colorectal cancer (13), non-small cell lung cancer (14), renal cell cancer (15) and osteosarcoma (16). YKL-40 may also be expressed within local inflammatory cells in inflamed tissues $(17,18)$ and it is considered to be a biomarker in inflammatory disease. The YKL-40 gene can regulate the extent of inflammation, and angiogenesis, and the process of inflammation resolution. In gynecologic malignant tumors, including ovarian cancer (19) and EC (20), YKL-40 serum levels were observed to be higher compared with healthy individuals. One previous study has have demonstrated that plasma YKL-40 protein levels are elevated in patients with EC and are associated with disease severity and prognosis (4). Previous studies also demonstrated that the expression of YKL-40 protein in EC tissue was associated with histology, stage and reduced survival time via immunohistochemistry $(20,21)$. YKL-40 gene and protein expression levels in cancerous tissue may determine the concentration in the blood. The serum YKL-40 level as a biomarker may have values in the diagnosis and prognosis of cancer.

YKL-40 mRNA may serve an important role in the proliferation, angiogenesis, and anti-apoptosis of cancer cells. A previous study reported potential biological functions and mechanisms of the YKL-40 gene within cancer cells. For example, in cholangiocarcinoma cells, YKL-40 mRNA may promote cell proliferation and migration by regulating the RAC- $\alpha$ serine/threonine-protein kinase/extracellular signal-regulated kinase (ERK) pathway (12). The YKL-40 protein has additionally been reported to promote tumor angiogenesis by inducing the ERK1/2 pathway (22). Research has demonstrated that the YKL-40 protein regulates the release of inflammatory cytokines and the activation of the mitogen-activated protein kinase signaling pathway 

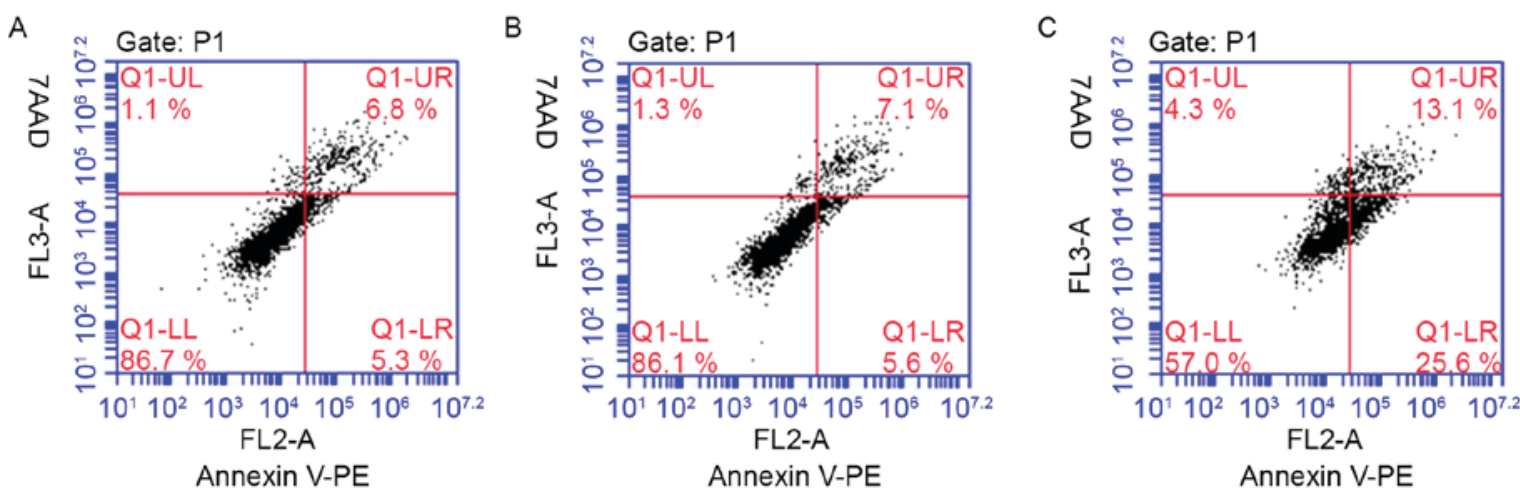

Figure 8. Flow cytometry analysis demonstrates the average cellular apoptosis rate in response to cisplatin, prior to and post-YKL-40 siRNA transduction via lentivirus. HEC-1A cells apoptosis rate increased following the inhibition of YKL-40 gene expression via si-YKL-40 transduction. (A) Blank, (B) Mock and (C) E.G cells. Blank, blank control group; Mock, mock-treatment group; E.G, experiment group; YKL-40, chitinase-3-like protein 1; si, small interfering; PE, phycoerythrin; 7AAD, 7-actinomycin D.

in colorectal carcinoma (23). A research has revealed that YKL-40 mRNA may inhibit ovarian cancer cell apoptosis via the MCL-1 BCL2 family apoptosis regulator gene (6). The YKL-40 protein may be induced by interleukin- 8 and tumor necrosis factor- $\alpha$ in colitis, and it promotes the development of colitis to tumor by regulating the nuclear factor- $\kappa \mathrm{B}$ pathway (24). In addition, YKL-40 mRNA also promotes macrophage recruitment and angiogenesis in colorectal cancer (23). A previous study reported that YKL-40 may regulate the sensitivity of a glioblastoma cell line to chemotherapy treatment via the signal transducer and activator of transcription 3 signaling pathway (25). These previous findings indicate that YKL-40 mRNA may have a role in cancer cells as an inflammatory factor. However, further investigation into the underlying mechanism is required.

RNA interference is a powerful tool for gene silencing. In astrocytoma, YKL-40 has been reported to induce angiogenesis and metastasis (2). In the present study, the expression of the YKL-40 gene within HEC-1A cells was significantly knocked down following transduction with a lentivirus. Additionally, YKL-40 gene activity was successfully inhibited by si-YKL-40; however, the same effect was not observed in the mock-treatment group. In cell proliferation assays, the proliferative ability of HEC-1A cells was inhibited by YKL-40 siRNA. In addition, the silencing of the YKL-40 gene within HEC-1A cells was associated with a reduction in the number of migrating and invading cells during the migration and Matrigel invasion assays. The number of migratory cells in the experimental group was significantly reduced compared with in the blank and the mock-treatment groups. These assays revealed that YKL-40 may control the migratory and invasive potential of HEC-1A cells.

Postoperative chemotherapy for residual tumor cells is an important and a primary treatment for advanced EC. At present, platinum drugs are widely used in a variety of first-line chemotherapy treatments; however, resistance to chemotherapy is a major limiting factor in the treatment of cancer, and EC is no exception. It is important to understand the underlying mechanism of EC chemotherapy drug resistance.

In the present study, the effects of cisplatin on the YKL-40 mRNA in human HEC-1A cells were investigated. The expression levels of YKL-40 mRNA within human HEC-1A cells were elevated following treatment with cisplatin for $48 \mathrm{~h}$. The results were consistent with those of van Linde et al (26); an ELISA revealed that serum YKL-40 levels were increased following chemotherapy in glioblastoma. YKL-40 is associated with chemotherapy sensitivity in drug-resistant tumor cell lines, and a reduction in the expression of YKL-40 in these cancer cell lines may increase the sensitivity of tumor chemotherapy drugs (25). However, a study of non-small cell lung cancer demonstrated that serum YKL-40 levels were downregulated following chemotherapy. Additionally, YKL-40 may be associated with the proliferation of EC and exerted an anti-apoptotic function when the HEC-1A cells were treated with chemotherapy drugs YKL-40 may serve an important role in the sensitivity to chemotherapy in certain cancer cells. In the present study, the effects of si-YKL-40 on the cisplatin sensitivity of EC HEC-1A cells $48 \mathrm{~h}$ post-treatment with cisplatin were determined via an MTT assay. The proliferative ability of the experimental group cells was significantly inhibited compared with the blank and mock-treatment groups when treated with $25 \mu \mathrm{mol} / 1$ cisplatin. This dosage of cisplatin was associated with the greatest sensitivity to chemotherapy exhibited by HEC-1A cells. FCM revealed that the average cellular apoptosis rate increased following the inhibition of YKL-40 gene expression within EC HEC-1A cells under the same cisplatin concentration $(25 \mu \mathrm{mol} / \mathrm{l})$. The proportion of apoptotic cells was significantly increased within the blank and mock-treatment groups compared with the experimental group. These assays demonstrated the association between the YKL-40 gene and cisplatin-based chemotherapy in HEC-1A cells. The YKL-40 gene may increase the sensitivity of cisplatin-based chemotherapy, while undertaking a mechanism of EC chemoresistance. Research into ovarian cancer has demonstrated that YKL-40 may act as an evaluation index to aid the prognosis of cancer chemotherapy (27). Cell migration assays revealed that si-YKL-40 inhibited the HEC-1A cell migration abilities, as well as chemoresistance in gliomas (28). These findings provide support for the hypothesis that the YKL-40 gene may serve an important role in the chemoresistant, proliferative and apoptotic abilities of EC.

A wealth of tumorigenic evidence from human cancer and animal tumor models indicates that elevated levels of angiogenic factors in cancer tissue correlate with tumor angiogenesis. 
Studies have investigated the inhibitory effects of YKL-40 gene on tumor growth and angiogenesis induced by YKL-40 siRNA (2). Other studies have demonstrated that the migratory and invasive abilities of ovarian cancer cells were inhibited by the silencing of YKL-40 gene, whereas YKL-40 overexpression may be associated with angiogenesis (6). In other malignancies (29-31), the proliferative, migratory and invasive abilities of cancer cells were inhibited by si-YKL-40 in vitro; tumor formation in the experimental group was reduced compared with the controlled group in vivo (2). In the present study, si-YKL-40 increased the sensitivity to cisplatin-based chemotherapy in EC HEC-1A cells. Biological behaviors, including proliferative, migration, invasive and anti-apoptotic abilities of HEC-1A cells were inhibited by YKL-40 gene RNA interference. In addition, the overexpression of YKL-40 may induce the proliferative, migratory and invasive abilities of EC cells, as in other tumors. Further investigation into the effects of YLK-40 and the underlying mechanism are required.

In conclusion, the results of the present study indicated that the expression of YKL-40 was effectively suppressed by si-YKL-40, which was associated with the inhibition of the biological behaviors of HEC-1A cells. YKL-40 siRNA increased the sensitivity of cisplatin-based chemotherapy in HEC-1A cells. Combining YKL-40 siRNA with other postoperative chemotherapies may provide more efficacious therapies for patients with EC. The YKL-40 gene may serve as a molecular target for the diagnosis and treatment of EC in the future. However, further clinical trials are required to understand the effects of YLK-40 in the management of EC.

\section{Acknowledgements}

Not applicable.

\section{Funding}

The present study was supported by the National Natural Scientific Foundation of China (grant no. 81360388) and the Natural Scientific Foundation of Guangxi Zhuang Autonomous Region, China (grant no. 2016GXNSFAA380258).

\section{Availability of data and materials}

All data generated or analysed during this study are included in this published article.

\section{Authors' contributions}

JF conceived the study and analyzed the data. LL and DL analyzed data. LL, YL, PS, CZ, XX and XH performed the experiments. LL wrote the manuscript. All authors read and approved the final manuscript.

\section{Ethics approval and consent to participate}

Not applicable.

\section{Consent for publication}

Not applicable.

\section{Competing interests}

The authors declare that they have no competing interests.

\section{References}

1. Brøchner CB, Johansen JS, Larsen LA, Bak M, Mikkelsen HB, Byskov AG, Andersen CY and Møllgård K: YKL-40 is differentially expressed in human embryonic stem cells and in cell progeny of the three germ layers. J Histochem Cytochem 60: 188-204, 2012.

2. Francescone RA, Scully S, Faibish M, Taylor SL, Oh D, Moral L, Yan W, Bentley B and Shao R: Role of YKL-40 in the angiogenesis, radioresistance, and progression of glioblastoma. J Biol Chem 286: 15332-15343, 2011

3. Lee CG, Hartl D, Lee GR, Koller B, Matsuura H, Da Silva CA, Sohn MH, Cohn L, Homer RJ, Kozhich AA, et al: Role of breast regression protein 39 (BRP-39)/chitinase 3-like-1 in Th2 and IL-13-induced tissue responses and apoptosis. J Exp Med 206: 1149-1166, 2009

4. Fan JT, Si XH, Liao Y and Shen P: The diagnostic and prognostic value of serum YKL-40 in endometrial cancer. Arch Gynecol Obstet 287: 111-115, 2013

5. Li LL, Fan JT, Li DH and Liu Y: Effects of a small interfering RNA targeting YKL-40 gene on the proliferation and invasion of endometrial cancer HEC-1A cells. Int J Gynecol Cancer 26: 1190-1195, 2016.

6. Chiang YC, Lin HW, Chang CF, Chang MC, Fu CF, Chen TC, Hsieh SF, Chen CA and Cheng WF: Overexpression of CHI3L1 is associated with chemoresistance and poor outcome of epithelial ovarian carcinoma. Oncotarget 6: 39740-39755, 2015.

7. Lax SF: Pathology of endometrial carcinoma. Adv Exp Med Biol 943: 75-96, 2017.

8. Dossus L, Allen N, Kaaks R, Bakken K, Lund E, Tjonneland A, Olsen A, Overvad K, Clavel-Chapelon F, Fournier A, et al: Reproductive risk factors and endometrial cancer: The European prospective investigation into cancer and nutrition. Int $\mathrm{J}$ Cancer 127: 442-451, 2010

9. Zhu Y, Shen J, Gao L and Feng Y: Estrogen promotes fat mass and obesity-associated protein nuclear localization and enhances endometrial cancer cell proliferation via the mTOR signaling pathway. Oncol Rep 35: 2391-2397, 2016.

10. Roslind A and Johansen JS: YKL-40: A novel marker shared by chronic inflammation and oncogenic transformation. Methods Mol Biol 511: 159-184, 2009.

11. Steponaitis G, Skiriutė D, Kazlauskas A, Golubickaitė I, Stakaitis R, Tamašauskas A and Vaitkienè P: High CHI3L1 expression is associated with glioma patient survival. Diagn Pathol 11: 42, 2016.

12. Thongsom S, Chaocharoen W,Silsirivanit A,WongkhamS,SripaB, Choe H, Suginta W and Talabnin C: YKL-40/chitinase-3-like protein 1 is associated with poor prognosis and promotes cell growth and migration of cholangiocarcinoma. Tumour Biol 37: 9451-9463, 2016.

13. Johansen JS, Christensen IJ, Jørgensen LN, Olsen J, Rahr HB, Nielsen KT, Laurberg S, Brünner N and Nielsen HJ: Serum YKL-40 in risk assessment for colorectal cancer: A prospective study of 4,496 subjects at risk of colorectal cancer. Cancer Epidemiol Biomarkers Prev 24: 621-626, 2015.

14. Wang XW, Cai CL, Xu JM, Jin H and Xu ZY: Increased expression of chitinase 3-like 1 is a prognosis marker for non-small cell lung cancer correlated with tumor angiogenesis. Tumour Biol 36: 901-907, 2015.

15. Vom Dorp F, Tschirdewahn S, Niedworok C, Reis H, Krause H, Kempkensteffen C, Busch J, Kramer G, Shariat SF, Nyirady P, et al: Circulating and tissue expression levels of YKL-40 in renal cell cancer. J Urol 195: 1120-1125, 2016.

16. Thorn AP, Daugaard S, Christensen LH, Christensen IJ and Petersen MM: YKL-40 protein in osteosarcoma tumor tissue. APMIS 124: 453-461, 2016.

17. Lai T, Chen M, Deng Z, L Y, Wu D, Li D and Wu B: YKL-40 is correlated with FEV1 and the asthma control test (ACT) in asthmatic patients: Influence of treatment. BMC Pulm Med 15: $1,2015$.

18. Gudmundsdottir S, Lieder R, Sigurjonsson OE and Petersen PH: Chitosan leads to downregulation of YKL-40 and inflammasome activation in human macrophages. J Biomed Mater Res A 103: 2778-2785, 2015 
19. Zou L, He X and Zhang JW: The efficacy of YKL-40 and CA125 as biomarkers for epithelial ovarian cancer. Braz J Med Biol Res 43: 1232-1238, 2010

20. Fan JT, Li MJ, Shen P, Xu H, Li DH and Yan HQ: Serum and tissue level of YKL-40 in endometrial cancer. Eur J Gynaecol Oncol 35: 304-308, 2014.

21. Kemik P, Saatli B, Yıldırım N, Kemik VD, Deveci B, Terek MC, Koçtürk S, Koyuncuoğlu M and Saygılı U: Diagnostic and prognostic values of preoperative serum levels of YKL-40, HE-4 and DKK-3 in endometrial cancer. Gynecol Oncol 140: 64-69, 2016.

22. Zhang W, Kawanishi M, Miyake K, Kagawa M, Kawai N, Murao K, Nishiyama A, Fei Z, Zhang X and Tamiya T: Association between YKL-40 and adult primary astrocytoma. Cancer 116: 2688-2697, 2010.

23. Kawada M, Seno H, Kanda K, Nakanishi Y, Akitake R, Komekado H, Kawada K, Sakai Y, Mizoguchi E and Chiba T: Chitinase 3-like 1 promotes macrophage recruitment and angiogenesis in colorectal cancer. Oncogene 31: 3111-3123, 2012.

24. Chen CC,Pekow J,Llado V, Kanneganti M,Lau CW, Mizoguchi A, Mino-Kenudson M, Bissonnette M and Mizoguchi E: Chitinase 3-like-1 expression in colonic epithelial cells as a potentially novel marker for colitis-associated neoplasia. Am J Pathol 179: 1494-1503, 2011.

25. Akiyama Y, Ashizawa T, Komiyama M, Miyata H, Oshita C, Omiya M, Iizuka A, Kume A, Sugino T, Hayashi N, et al: YKL-40 downregulation is a key factor to overcome temozolomide resistance in a glioblastoma cell line. Oncol Rep 32: 159-166, 2014.
26. van Linde ME, van der Mijn JC, Pham TV, Knol JC, Wedekind LE, Hovinga KE, Aliaga ES, Buter J, Jimenez CR, Reijneveld JC and Verheul HM: Evaluation of potential circulating biomarkers for prediction of response to chemoradiation in patients with glioblastoma. J Neurooncol 129: 221-230, 2016.

27. Boisen MK, Madsen CV, Dehlendorff C, Jakobsen A, Johansen JS and Steffensen KD: The prognostic value of plasma YKL-40 in patients with chemotherapy-resistant ovarian cancer treated with bevacizumab. Int J Gynecol Cancer 26: 1390-1398, 2016.

28. Ku BM, Lee YK, Ryu J, Jeong JY, Choi J, Eun KM, Shin HY, Kim DG, Hwang EM, Yoo JC, et al: CHI3L1 (YKL-40) is expressed in human gliomas and regulates the invasion, growth and survival of glioma cells. Int J Cancer 128: 1316-1326, 2011.

29. Jefri M, Huang YN, Huang WC, Tai CS and Chen WL: YKL-40 regulated epithelial-mesenchymal transition and migration/invasion enhancement in non-small cell lung cancer. BMC Cancer 15: 590,2015

30. Mylin AK, Abildgaard N, Johansen JS, Heickendorff L, Kreiner S, Waage A, Turesson I and Gimsing P; Nordic Myeloma Study Group: Serum YKL-40: A new independent prognostic marker for skeletal complications in patients with multiple myeloma. Leuk Lymphoma 56: 2650-2659, 2015.

31. Jeet V, Tevz G, Lehman M, Hollier B and Nelson C: Elevated YKL40 is associated with advanced prostate cancer (PCa) and positively regulates invasion and migration of PCa cells. Endocr Relat Cancer 21: 723-737, 2014. 